\title{
Neutrinoless double beta decay, neutrino mass and bilinear R-parity breaking supersymmetry
}

\author{
M. Hirsch ${ }^{\mathrm{a}}$ \\ ${ }^{a}$ Instituto de Física Corpuscular - C.S.I.C. \\ Departamento de Física Teòrica, Universitat of València, \\ Edificio Institutos de Paterna, Apartado de Correos 2085 \\ 46071 València, Spain \\ mahirsch@flamenco.ific.uv.es
}

Neutrinoless double beta $\left(\beta \beta_{0 \nu}\right)$ decay violates lepton number; its absence stringently constrains the parameters of theories beyond the standard model in which the neutrino has a Majorana mass. R-parity violating weak-scale supersymmetry is a prominent example of such models. Double beta decay in supersymmetry with explicit bilinear $\mathrm{R}$-parity breaking is discussed and current limits on the $\beta \beta_{0 \nu}$ decay half life of ${ }^{76} \mathrm{Ge}$ are used to extract upper bounds on the R-parity breaking parameters of the first generation. Moreover, it is shown that the effective Majorana neutrino mass, measured in $\beta \beta_{0 \nu}$ decay, is non-zero once the 1-loop corrections are taken into account even for the case of perfect alignment $\left(\Lambda_{i}:=\left(\left\langle\tilde{\nu}_{i}\right\rangle \mu-v_{1} \epsilon_{i}\right) \equiv 0\right)$ among the R-parity violating parameters.

\section{Introduction}

Neutrinoless double beta $\left(\beta \beta_{0 \nu}\right)$ decay is a $\Delta L=2$ process and therefore one naturally expects it to occur in models with lepton number violation in the Lagrangian. Even though there is a variety of mechanisms inducing $\beta \beta_{0 \nu}$ decay in gauge theories, one can show that whatever the leading mechanism is at least one of the neutrinos will be a Majorana particle [1], as illustrated in the black-box diagram of Fig. 1. This well-known argument establishes a deep connection between Majorana neutrino masses and $\beta \beta_{0 \nu}$ decay: in gauge theories one can not occur without the other being present. The same remains true in supersymmetric theories [2], where moreover one can show that also the supersymmetric partner of the neutrino must have a $B-L$ violating Majorana-like mass term, if a Majorana mass of the neutrino exists [2]. Turning the argument around, one expects that the observed absence of $\beta \beta_{0 \nu}$ decay allows to derive stringent limits on $\not_{p}$ parameters. This has been shown for models with explicit trilinear R-parity breaking in [3, 4 ] and for SUSY with explicit bilinear R-parity breaking in [5.6]. Here, I will mainly report on the results derived in $[5]$.
Section 2 sets up the notation and definitions of the bilinear R-parity breaking model, whereas section 3 discusses $\beta \beta_{0 \nu}$ decay in bilinear $\not R_{p}$ SUSY at tree level. In section 4 , the $\beta \beta_{0 \nu}$ decay in bilinear $R_{p}$ SUSY is discussed at the level of 1-loop for the first time in literature.

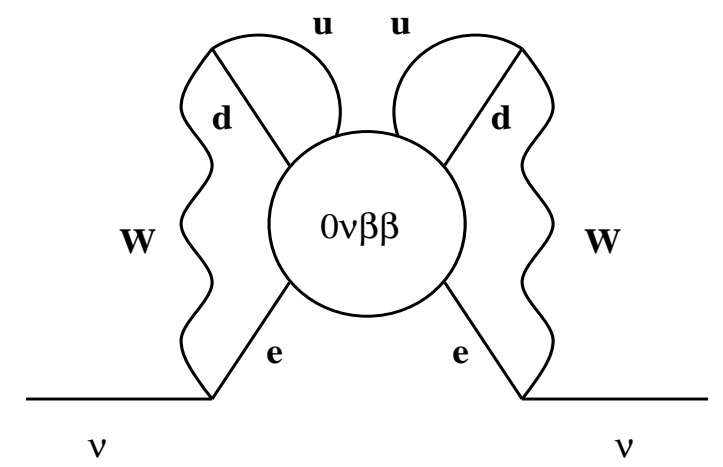

Figure 1: Diagram illustrating the connection between the Majorana mass of the neutrino and the amplitude of double beta decay.

\section{Minimal R-parity broken supersymme- try}

The minimal supersymmetric extension of the standard model (MSSM) has a conserved R- 
parity. This is a multiplicative quantum number which can be defined as $R_{P}=(-1)^{3 B+L+2 S}$, where $B$ and $L$ are the baryon and lepton number and $S$ the spin of the corresponding particle. This property of the MSSM [7] is theoretically ad hoc since the origin of R-parity conservation is unknown.

In supersymmetry there is actually no distinction between the lepton doublet and the Higgs doublet superfield giving mass to the down-type quarks unless conservation of lepton number is assumed. This fact can be accounted for by defining a superfield $\hat{\Phi}$ as

$\hat{\Phi}=\left(\hat{H}_{1}, \hat{L}_{1}, \hat{L}_{2}, \hat{L}_{3}\right)$.

For the MSSM field content the most general gauge invariant form of the renormalizable superpotential can then be written as

$$
\begin{aligned}
W & =\epsilon_{a b}\left[\lambda_{e}^{I J k} \hat{\Phi}_{I}^{a} \hat{\Phi}_{J}^{b} \hat{E}_{k}^{C}+\lambda_{d}^{I j k} \hat{\Phi}_{I}^{a} \hat{Q}_{j}^{b} \hat{D}_{k}^{C}\right. \\
& \left.+h_{u}^{j k} \hat{Q}_{j}^{a} \hat{H}_{2}^{b} \hat{U}_{k}^{C}+\mu^{I} \hat{\Phi}_{I}^{a} \hat{H}_{2}^{b}\right] .
\end{aligned}
$$

Here, $\hat{Q}$ and $\hat{D}^{C}, \hat{U}^{C}$ are the quark doublet and singlets superfields, respectively, $\hat{E}^{C}$ is the lepton singlet superfield and $\hat{H}_{2}$ the Higgs superfields with $Y\left(\hat{H}_{2}\right)=1$ responsible for the up-type quark masses, with $h_{u}^{j k}$ being the corresponding Yukawa couplings. The indices $j, k=1,2,3$ denote generations, whereas $I, J=0,1,2,3$. The indices $a, b$ are $S U(2)$ indices. The part of $W$ with indices $I, J=0$ corresponds to the MSSM superpotential, wheras $I, J=1,2,3$ represents the R-parity violating terms. The vector $\mu^{I}$ is $\mu^{I}=\left(\mu, \epsilon_{e}, \epsilon_{\mu}, \epsilon_{\tau}\right)$.

If the only source of RPV in the model was found in the superpotential one could easily rotate the field $\hat{\Phi}$ into a basis $\hat{\Phi}^{\prime}$ with $\mu^{I^{\prime}}=$ $\left(\mu^{\prime}, 0,0,0\right)$ effectively eliminating the bilinear terms. However, another source of RPV is found in the soft supersymmetry breaking part of the scalar potential. It contains the terms:

$$
\begin{aligned}
V_{R_{p}}^{\text {soft }} & =\tilde{A}_{e}^{I J k} \tilde{\Phi}_{I} \tilde{\Phi}_{J} \tilde{E}_{k}^{C}+\tilde{A}_{d}^{I j k} \tilde{\Phi}_{I} \tilde{Q}_{j} \tilde{D}_{k}^{C} \\
& +B^{I} \tilde{\Phi}_{I} H_{2}+\left(m_{I J}^{2}+\mu_{I} \mu_{J}\right) \tilde{\Phi}_{I} \tilde{\Phi}_{J}^{\dagger}+\cdots
\end{aligned}
$$

where the dots represent terms not interesting for the discussion here. Rotating the superpotential as discussed above, it is easy to see that as long as the $B^{I}$ are not exactly parallel to the $\mu^{I}$ the effects of the bilinear terms of the superpotential in the rotated basis will reappear in the soft SUSY breaking terms.

The presence of the bilinear terms in (3) imply that in general the sneutrino fields acquire Vevs and as a result the leptons and gauginos of the model mix. The neutralino mass matrix which in the MSSM is a $(4 \times 4)$ matrix in the bilinear $\not R_{p}$ MSSM is a $(7 \times 7)$ matrix, including in addition the three generations of neutrinos. It can be written in the following form:

$\mathcal{M}_{0}=\left(\begin{array}{cc}0 & m \\ m^{T} & \mathcal{M}_{\chi^{0}}\end{array}\right)$.

Here, the submatrix $m$ contains entries from the bilinear $\not R_{p}$ parameters,

$m=\left(\begin{array}{cccc}-\frac{1}{2} g^{\prime} \omega_{e} & \frac{1}{2} g \omega_{e} & 0 & -\epsilon_{e} \\ -\frac{1}{2} g^{\prime} \omega_{\mu} & \frac{1}{2} g \omega_{\mu} & 0 & -\epsilon_{\mu} \\ -\frac{1}{2} g^{\prime} \omega_{\tau} & \frac{1}{2} g \omega_{\tau} & 0 & -\epsilon_{\tau}\end{array}\right)$,

$\omega_{i}:=\left\langle\tilde{\nu}_{i}\right\rangle . \mathcal{M}_{\chi^{0}}$ is the MSSM neutralino mass matrix given by,

$\mathcal{M}_{\chi^{0}}=\left(\begin{array}{cccc}M_{1} & 0 & -\frac{1}{2} g^{\prime} v_{1} & \frac{1}{2} g^{\prime} v_{2} \\ 0 & M_{2} & \frac{1}{2} g v_{1} & -\frac{1}{2} g v_{2} \\ -\frac{1}{2} g^{\prime} v_{1} & \frac{1}{2} g v_{1} & 0 & -\mu \\ \frac{1}{2} g^{\prime} v_{2} & -\frac{1}{2} g v_{2} & -\mu & 0\end{array}\right)$

It is interesting to note that the matrix (4i) has such a texture that at tree level only one of the three neutrinos gets massive, leaving two massless but mixed states in the spectrum.

If the $R_{p}$ parameters are small in the sense that for

$\xi=m \cdot \mathcal{M}_{\chi^{0}}^{-1}$

all $\xi_{i j} \ll 1$, one can find an approximate solution for the neutrino/neutralino mass matrix.

In leading order in $\xi$ the mixing matrix $\Xi$ which diagonalizes the mass matrix is given by,

$$
\begin{aligned}
\Xi^{*} & =\left(\begin{array}{cc}
V_{\nu}^{T} & 0 \\
0 & N^{*}
\end{array}\right)\left(\begin{array}{cc}
1-\frac{1}{2} \xi \xi^{\dagger} & -\xi \\
\xi^{\dagger} & 1-\frac{1}{2} \xi^{\dagger} \xi
\end{array}\right) \\
& =\left(\begin{array}{cc}
V_{\nu}^{T}\left(1-\frac{1}{2} \xi \xi^{\dagger}\right) & -V_{\nu}^{T} \xi \\
N^{*} \xi^{\dagger} & N^{*}\left(1-\frac{1}{2} \xi^{\dagger} \xi\right)
\end{array}\right) \quad(7)
\end{aligned}
$$


The second matrix in eq. (7) above blockdiagonalizes $\mathcal{M}_{0}$ approximately to the form $\operatorname{diag}\left(m_{e f f}, \mathcal{M}_{\chi^{0}}\right)$, where

$$
\begin{aligned}
& m_{\text {eff }}=-m \cdot \mathcal{M}_{\chi^{0}}^{-1} m^{T} \\
= & \frac{M_{1} g^{2}+M_{2} g^{\prime 2}}{4 \operatorname{det}\left(\mathcal{M}_{\chi^{0}}\right)}\left(\begin{array}{ccc}
\Lambda_{e}^{2} & \Lambda_{e} \Lambda_{\mu} & \Lambda_{e} \Lambda_{\tau} \\
\Lambda_{e} \Lambda_{\mu} & \Lambda_{\mu}^{2} & \Lambda_{\mu} \Lambda_{\tau} \\
\Lambda_{e} \Lambda_{\tau} & \Lambda_{\mu} \Lambda_{\tau} & \Lambda_{\tau}^{2}
\end{array}\right)
\end{aligned}
$$

Here, $\operatorname{det}\left(\mathcal{M}_{\chi^{0}}\right)$ is the determinant of $\mathcal{M}_{\chi^{0}}$ and

$\Lambda_{i}=\mu \omega_{i}-v_{1} \epsilon_{i}$.

$\vec{\Lambda}:=\left(\Lambda_{e}, \Lambda_{\mu}, \Lambda_{\tau}\right)$, which will be called the alignment vector, plays a very prominent role in the bilinear $R_{p}$ model. As one can read off from eq. (8) at tree level neutrinos are massless if $\vec{\Lambda} \equiv 0$. Second, even though two neutrinos are massless at tree level, all neutrinos mix with each other and the heavy states, except if (and only if) the corresponding component $\Lambda_{i}$ vanishes.

\section{3. $\beta \beta_{0 \nu}$ decay in the bilinear $R_{p}$ MSSM at tree level}

In the bilinear $\not R_{p}$ MSSM there are 4 Feynman diagrams in lowest order of perturbation, see fig. 2. One can show, however, that the simplest of these graphs, the neutrino mass contribution is always dominant [5].

The double beta decay observable, under the assumption that the $\not R_{p}$ parameters are small, can be written as

$$
\left\langle m_{\nu}\right\rangle=\sum_{j}^{\prime} U_{e j}^{2} m_{j}=\frac{2}{3} \frac{g^{2} M_{2}}{\operatorname{det}\left(\mathcal{M}_{\chi^{0}}\right)} \Lambda_{e}^{2}
$$

where $\Lambda_{e}=\omega_{e} \mu-v_{1} \epsilon_{e}$ and the prime indicates summation over only light fermion states Note, that eq. (10) is proportional to the alignment factor $\Lambda_{e}$, i.e. at tree level double beta decay would vanish, as is the case for the neutrino mass, in the limit of $\vec{\Lambda} \equiv 0$. Using the experimentally measured lower limit on the half life of $\beta \beta_{0 \nu}$ decay, Eq. (10) can then be used to establish limits on $\Lambda_{e}$ as a function of the R-parity conserving SUSY parameters $\mu, M_{2}$ and $\tan \beta$. An example is shown in fig. 3 . The small region(s) extending to the upper right are the alignment regions were $\Lambda_{e} \rightarrow 0$. Along these lines there is no constraint on $\left(\epsilon_{e}, \omega_{e}\right)$ from $\beta \beta_{0 \nu}$ decay at tree-level.

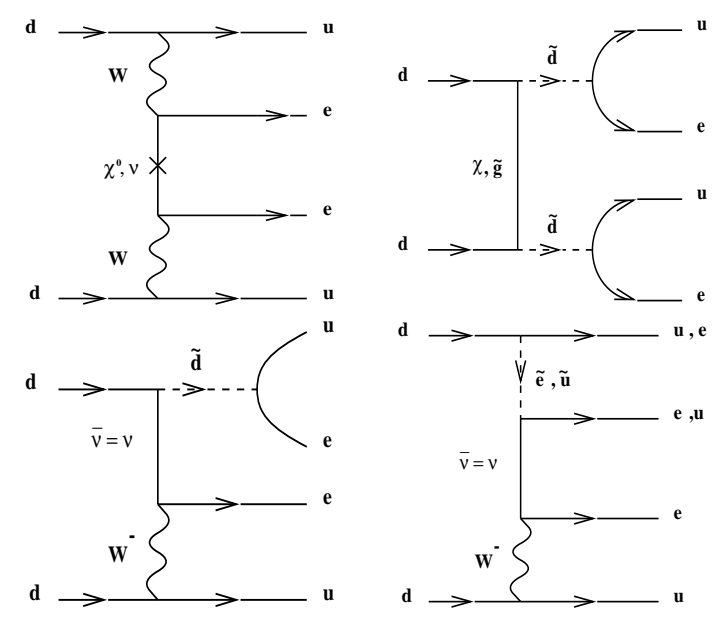

Figure 2: Leading order Feynman diagrams in the bilinear $R_{p}$ model.

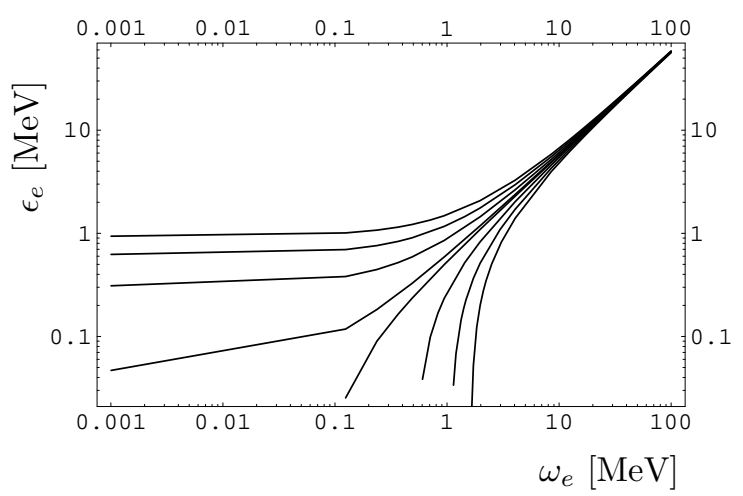

Figure 3: Excluded ranges in plane $\left(\epsilon_{e}, \omega_{e}\right)$ using $\left\langle m_{\nu}\right\rangle \leq 0.5 \mathrm{eV}$ for $\tan \beta=1$ and $\mu=$ $100 \mathrm{GeV}$, for different values of $M_{2}, M_{2}=$ $100,200,500,1000 \mathrm{GeV}$. Note that the allowed range is always in between two lines of constant $M_{2}$.

Another way of visualizing the constraints is to invert the procedure and calculate the theoretically expected half lives as a function of, for example $\omega_{e}$. The results of such a study is shown in figure 4 . The experimental limit is taken from 
[8]. Values of $\omega_{e}$ larger than about $2 \mathrm{MeV}$ are not allowed, as long as $\omega_{e}$ and $\epsilon_{e}$ are not perfectly aligned.

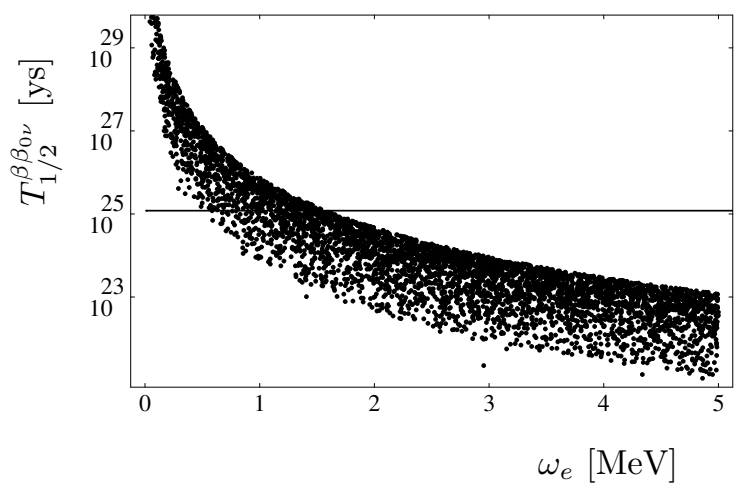

Figure 4: Calculated half-life for the $\beta \beta_{0 \nu}$ decay of ${ }^{76} \mathrm{Ge}$ as function of $\omega_{e}$ for a random variation of the MSSM parameters, $M_{2}$ and $\mu$ from $100 \mathrm{GeV}$ to $1 \mathrm{TeV}$ and $\tan \beta=1-50$.

\section{Some results for $\beta \beta_{0 \nu}$ decay at 1-loop}

Since double beta decay in the bilinear model in the lowest order of perturbation is strictly proportional to $\Lambda_{e}$, it is an interesting question to ask, whether it is possible to determine an absolute upper bound on individual parameters, $\epsilon_{e}$ (and $\omega_{e}$ ) even for the special case $\Lambda_{e} \equiv 0$, going to higher orders of perturbation.

There are three simple topologies of relevant Feynman diagrams contributing to the neutrinoneutralino mass matrix at 1-loop [9,10]. With these the one-loop corrected mass matrix is calculated as,

$$
\begin{aligned}
M_{i j}^{\text {pole }} & =M_{i j}^{\overline{D R}}\left(\mu_{R}\right)+\frac{1}{2}\left(\Pi_{i j}\left(p_{i}^{2}\right)+\Pi_{i j}\left(p_{j}^{2}\right)\right. \\
& \left.-m_{\chi_{i}^{0} \Sigma_{i j}}\left(p_{i}^{2}\right)-m_{\chi_{j}^{0}} \Sigma_{i j}\left(p_{j}^{2}\right)\right)
\end{aligned}
$$

where $\Sigma_{i j}$ and $\Pi_{i j}$ are self-energies. For a complete description see 10 . Here, $\overline{D R}$ signifies the minimal dimensional reduction subtraction scheme and $\mu_{R}$ is the renormalization scale.

Although the 1-loop corrections for the heavy states ("neutralinos") can be expected to be moderate, for the two lightest states they are essential. Moreover, the mixing of the neutrinos to the down type higgsinos and to the charged higgsino contains a piece which is directly proportional to $\epsilon_{i}$ and not only to $\Lambda_{i}$ [5, 10]. One therefore has to expect, that after inclusion of the 1-loop correction $\left\langle m_{\nu}\right\rangle$ will be sensitive to the value of $\epsilon_{e}$ directly.

Following the procedure of [9, 10] I have calculated values for the 1-loop neutrino mass matrix and deduced $\left\langle m_{\nu}\right\rangle$ for several $10^{3}$ randomly generated points in SUSY parameter space. Results are shown in fig. 5. The ranges of parameters were chosen as follows. For the MSSM parameters: $|\mu|, M_{2} \leq 500 \mathrm{GeV}$, the common scalar mass $m_{0}=0.2-1 \mathrm{TeV}, \tan \beta=2.5-20$. Since double beta decay is sensitive only to the first generation $R_{p}$ parameters, it suffices to state that in generating the plot I have always kept $\Lambda_{e}$ at least a factor of 100 smaller than required by the tree level bound. The last condition is applied to guarantee that only the highly aligned part of parameter space is explored.

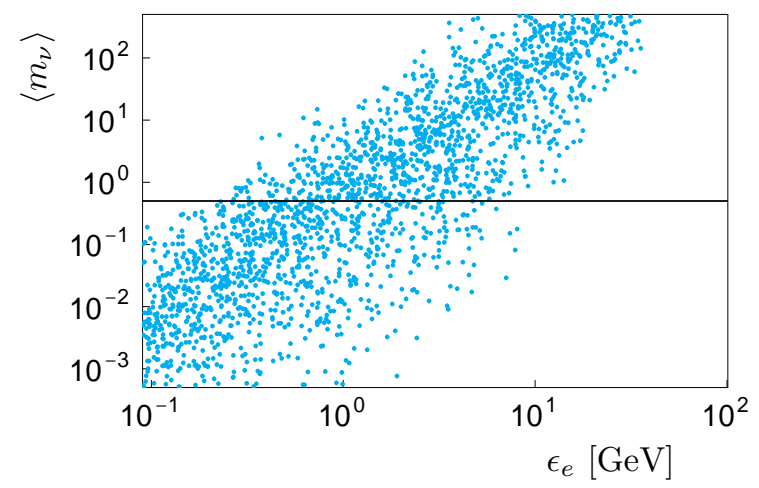

Figure 5: Calculated values of $\left\langle m_{\nu}\right\rangle$ including the 1-loop corrections, as a function of $\epsilon_{e}$, for $\Lambda_{e} \leq 10^{-2} \times \Lambda_{e}^{\text {tree,max }} . \Lambda_{e}^{\text {tree, } \max }$ is the maximal $\Lambda_{e}$ allowed from the tree-level analysis. The horizontal line indicates the current experimental upper limit.

Because of the complexity of the 1-loop calculation it is impossible to derive a semi-analytical bound as in the tree level case. Nevertheless, figure 5 shows that $\epsilon_{e}$ can not be larger than about $5-10 \mathrm{GeV}$. Note, that larger values of $m_{0}$ would allow only for very slightly larger values of $\epsilon_{e}$, 
whereas larger values for $\tan \beta$ would give lower upper limits.

It is also interesting to ask how reliable the tree-level estimation for the double beta decay observable is. Following the arguments about the alignment, discussed above, it is expected that if $\epsilon_{e}^{2} / \Lambda_{e}$ is small, i.e. the suppression of $\Lambda_{e}$ is due to the smallness of $\epsilon_{e}$ and $\omega_{e}$ themselves and not due to cancellations among the two terms in $\Lambda_{e}$, the tree level expression should be a good approximation, wheras for large $\epsilon_{e}^{2} / \Lambda_{e}$ one expects the tree level estimate to fail badly. This is demonstrated in fig. 6, which nicely confirms this qualitative expectation. Note, however, that even for $\epsilon_{e}^{2} / \Lambda_{e}$ as small as (few) $10^{-2}$ the tree-level expression is not reliable and it certainly fails if $\epsilon_{e}^{2} / \Lambda_{e}$ is larger than order $\mathcal{O}(1)$.

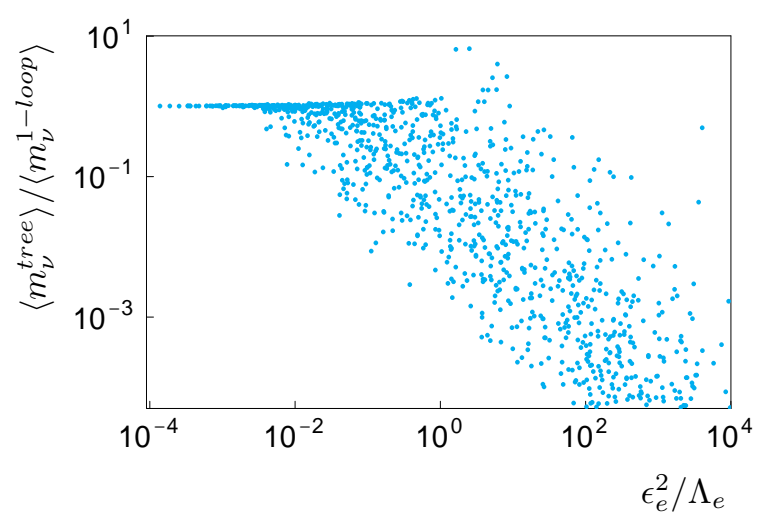

Figure 6: Calculated ratios of $\left\langle m_{\nu}\right\rangle$ at treelevel to $\left\langle m_{\nu}\right\rangle$ including the 1-loop corrections, as a function of $\epsilon_{e}^{2} / \Lambda_{e}$, see text.

\section{Summary}

I have discussed the contributions of (bilinear) R-parity breaking supersymmetry to neutrinoless double beta decay. $\beta \beta_{0 \nu}$ decay constrains only a subset of the possible bilinear parameters, namely $\epsilon_{e}$ and the sneutrino VEV of the first generation $\omega_{e}$. This is a general property of the theory and does not mean any fine-tuning of parameters. For the first generation $R_{p}$ parameters, on the other hand, $\beta \beta_{0 \nu}$ decay provides very stringent limits, typically of the order of a few hundred $\mathrm{keV}$ up to a few $\mathrm{MeV}$.

Interesting is the fact that $\beta \beta_{0 \nu}$ decay is strictly proportional to the alignment factor $\Lambda_{e}$ only at tree level. Once one goes to the next order in perturbation theory, $\beta \beta_{0 \nu}$ is sensitive to $R_{p}$ parameters even in the case of perfect alignment. Although the limits in this special parameter range are less stringent than in the non-aligned case by about 3 orders of magnitude, they nevertheless require that $\epsilon_{e} / \mu \leq 0.01$, i.e. even for perfect alignment R-parity violation can not be maximal.

\section{Acknowledgement}

I like to thank J.W.F. Valle for his always enthusiastic collaboration, S.G. Kovalenko for useful discussions and J.C. Romão for his help with the 1-loop calculations. This work was supported by the Spanish DGICYT under grant PB95-1077 and by the European Union's TMR program under grants ERBFMRXCT960090 and ERBFMBICT983000.

\section{REFERENCES}

1. J. Schechter and J.W.F. Valle, Phys.Rev. D 25 (1982) 2951

2. M. Hirsch, H.V. Klapdor-Kleingrothaus and S.G. Kovalenko, Phys. Lett. B398 (1997) 311; ibid B403 (1997) 291

3. M. Hirsch, H.V. Klapdor-Kleingrothaus and S.G. Kovalenko, Phys. Rev. Lett. 75 (1995) 17; Phys. Rev. D53 (1996) 1329; Phys. Lett. B372 (1996) 181, erratum B381 (1996) 488

4. A. Faessler, S.G. Kovalenko, F. Simkovic and J. Schwieger, Phys. Rev. Lett. 78 (1997) 183

5. M. Hirsch and J.W.F. Valle, hep-ph/9812463, Nucl. Phys. B (1999) in press

6. A. Faessler, S. Kovalenko, F. Simkovic, Phys.Rev. D57 (1998) 055004 hep$\mathrm{ph} / 9712535$

7. For a review on the MSSM, see for example: H.E. Haber and G.L.Kane, Phys. Rep. 117 (1985) 75

8. L. Baudis et al., Phys. Lett. B407 (1997) 219

9. J.C. Romão, M.A. Diaz, M. Hirsch, W. Porod and J.W.F. Valle, hep-ph/9907499

10. J.C. Romão, M.A. Diaz, M. Hirsch, W. Porod and J.W.F. Valle, in preperation 